\title{
Metastatic testicular germ cell tumor with left atrial metastasis via the right pulmonary vein
}

\author{
Anish Adhikari ${ }^{1}$, Suchet Kumar ${ }^{1}$, and Steven Fein ${ }^{2}$ \\ ${ }^{1}$ Albany Medical Center \\ ${ }^{2}$ Albany Medical Center Hospital
}

June 16, 2020

\begin{abstract}
We present a 37-year-old man with history of incompletely treated testicular germ cell tumor presenting with persistent cough. Computed tomography $(\mathrm{CT})$ demonstrated a large pulmonary mass invading the mediastinal vasculature, bronchi, and thrombus in the pulmonary vein extending into the left atrium. Biopsy of the lung mass confirmed germ cell tumor. Transthoracic echocardiography revealed a mass in the left atrium, consistent with a tumor or thrombus. Systemic chemotherapy was started with cisplatin and etoposide with curative intent.
\end{abstract}

\section{Keywords}

Echocardiography, atrial mass, germ cell, testicular cancer

\section{Introduction}

Germ cell testicular tumors causing left atrial mass via the pulmonary vein are rare, with few case reports in the literature. Cardiac metastasis is seen in $9.1 \%$ of all patients with malignancy on post-mortem. Most common causes of cardiac metastasis in order are lung, breast, and ovarian malignancy. ${ }^{1}$ Recognition of cardiac involvement is imperative as complications including stroke ${ }^{2}$, acute limb ischemia ${ }^{3}$ and pulmonary venous obstruction ${ }^{4}$ may occur.

Transthoracic echocardiography (TTE) was used to further investigate the extent of spread of a mass seen on computed tomography $(\mathrm{CT})$.

\section{Case report}

A 37-year-old man presented with persistent cough for several weeks. He denied dyspnea, orthopnea, peripheral edema, syncope. He had a history of right testicular germ cell tumor, for which he had undergone orchiectomy seven months prior to presentation. He was lost to follow up and never started systemic therapy as planned. Physical exam was unremarkable except for evidence of right orchiectomy. Chest x-ray revealed multiple lung masses. He subsequently underwent a contrast enhanced CT of the chest. CT (image 1) showed a right pulmonary vein filling defect extending into the left atrium, large right lung mass $15.4 \mathrm{~cm}$ in greatest dimension extending into the right hilum and mediastinum with invasion of the right mainstem bronchus, the pulmonary veins, and encasement of the right main pulmonary artery and the branches to the right upper lower and middle lobes.

Image 1. Coronal view of right lung tumor demonstrating the size of the tumor. Tumor is invading the bronchus and mediastinum.

Histology from a CT guided biopsy of the mass confirmed mixed germ cell tumor. 
Image 2. Left atrial (LA) mass (arrow).

A TTE was done to further characterize the left atrial mass (image 2). A large mass which extends $4 \mathrm{~cm}$ from superior aspect of LA into LA cavity, and is $1 \mathrm{~cm}$ in diameter is identified. The mitral valve leaflets were normal with no evidence of regurgitation or stenosis.

Neo-adjuvant cisplatin and etoposide systemic chemotherapy was started with curative intent, he is planned for four cycles. Surgical resection of the tumor after chemical cytoreduction is being considered. Due to the unique situation posed by the COVID 19 pandemic, he was unable to obtain pre-treatment pulmonary function testing. Hence, bleomycin was omitted. He was started on anticoagulation with unfractionated heparin drip to minimize risk of embolization from the mass. His treatment course was complicated by new onset paroxysmal atrial fibrillation with rapid ventricular response, which was controlled with metoprolol.

\section{Discussion}

We present a 37-year-old man with metastatic germ cell tumor with tumor extending into the left atrium. He did not receive the planned adjuvant chemotherapy following orchiectomy, as he was lost to follow up. This may have played a role in developing extensive metastatic disease, as he had no evidence of macroscopic metastatic disease when he had orchiectomy seven months prior to presentation. The unique features of our case include the large size of the tumor, extensive involvement of multiple mediastinal structures, and hematogenous, intracavitary spread to the left atrium. We postulate the atrial fibrillation may have arisen due to irritation of the pulmonary vein by the tumor.

Cardiac metastasis arising from testicular germ cell tumors is rare.$^{5}$ Only twenty cases of testicular metastatic tumor extending from the pulmonary vein into any cardiac chamber have been reported in the literature. Of these, only four cases had left atrial involvement. Three case reports described patients with rapidly progressive disease who died within six months of diagnosis. ${ }^{3,5} \mathrm{~A}$ favorable outcome was seen in a Turkish patient who had surgical resection of the left atrial mass. ${ }^{6}$

The mechanism for intracavitary spread to the left atrium can be hematogenous via the pulmonary vein, ${ }^{7}$ lymphatic, or contiguous spread from surrounding structures. ${ }^{8}$

Given the widely variable prognosis of testicular versus pulmonary metastatic disease, identifying the primary site of the malignancy can radically change treatment goals from palliative to curative intent.

Compared to testicular cancer, advanced lung malignancy is a more common cause of intracavitary metastatic disease. A retrospective analysis showed $0.5 \%$ of advanced lung malignancy spread to the left atrium via the pulmonary vein. ${ }^{9}$ The five-year survival rate for metastatic lung carcinoma was $5.8 \%$ according to the Surveillance, Epidemiology, and End Result database (SEER) between 2010-2016. ${ }^{10}$ The SEER database showed the five year survival for distant metastatic testicular cancer was $72.5 \%$ between $2010-2016 .{ }^{10}$ There is not enough data to determine if cardiac metastasis portends a worse prognosis than metastasis to other site.

\section{Conclusion}

Left atrial metastasis from a testicular tumor is rare. To our knowledge, this is the first case of such a large tumor with invasion of multiple mediastinal structures, including the left atrium. If a left atrial mass is identified on TTE, a thorough search for a primary source beyond the lung must be conducted. TTE is a non-invasive, readily available option for further characterizing cardiac masses seen on CT. There is risk of embolism, tamponade, arrhythmia, and outflow obstruction with testicular cardiac metastasis. ${ }^{5}$ The risks associated with left atrial mass and the overall favorable prognosis of testicular tumors makes identifying these masses and their source important for guiding management and reducing risk of complications.

\section{References:}

1. Bussani R, De-Giorgio F, Abbate A, Silvestri F. Cardiac metastases. Journal of clinical pathology. 2007;60(1):27-34. 
2. Ballo P, Laureano R, Briganti M, et al. Left Atrial Mass Invasion from Pulmonary Neoplasm Extension via the Right Upper Pulmonary Vein Presenting as Ipsilateral Stroke. Case reports in medicine.2016;2016:7084234.

3. Singh A, Jenkins DP, Dahdal M, Dhar S, Ratnatunga CP. Recurrent arterial embolization from a metastatic germ cell tumor invading the left atrium. The Annals of thoracic surgery.2000;70(6):2155-2156.

4. Liaw CC, Chang H, Yang TS, Wen MS. Pulmonary Venous Obstruction in Cancer Patients. Journal of oncology. 2015;2015:210916.

5. Nunes MCP, Moreira DR, Ferrari TCA. Cardiac metastasis from yolk sac tumor: case report and review. Experimental Hematology \&3 Oncology. 2013;2(1):13.

6. Huseyin S, Yüksel V, Okyay A, et al. A rare cause in etiology of left atrial mass: metastatic testicular germ cell tumor. Kardiochir Torakochirurgia Pol. 2016;13(1):45-48.

7. Desai MY, Mankad S. Extension of Bronchogenic Carcinoma Through Pulmonary Vein into the Left Atrium Detected by Echocardiography.Echocardiography. 2004;21(2):189-191.

8. Ucak A, Inan K, Onan B, Temizkan V, Alp I, Yilmaz AT. Free-Floating Tumor Thrombus in the Left Atrium Associated with Non-Small Cell Lung Cancer. 2009;24(6):686-689.

9. Riquet M, Grand B, Arame A, et al. Lung cancer invading the pericardium: quantum of lymph nodes. The Annals of thoracic surgery. 2010;90(6):1773-1777.

10. Howlader N NA, Krapcho M, Miller D, Brest A, Yu M, Ruhl J, Tatalovich Z, Mariotto A, Lewis DR, Chen HS, Feuer EJ, Cronin KA SEER Cancer Statistics Review. 1975-2017; November 2019. Accessed May 2020 .

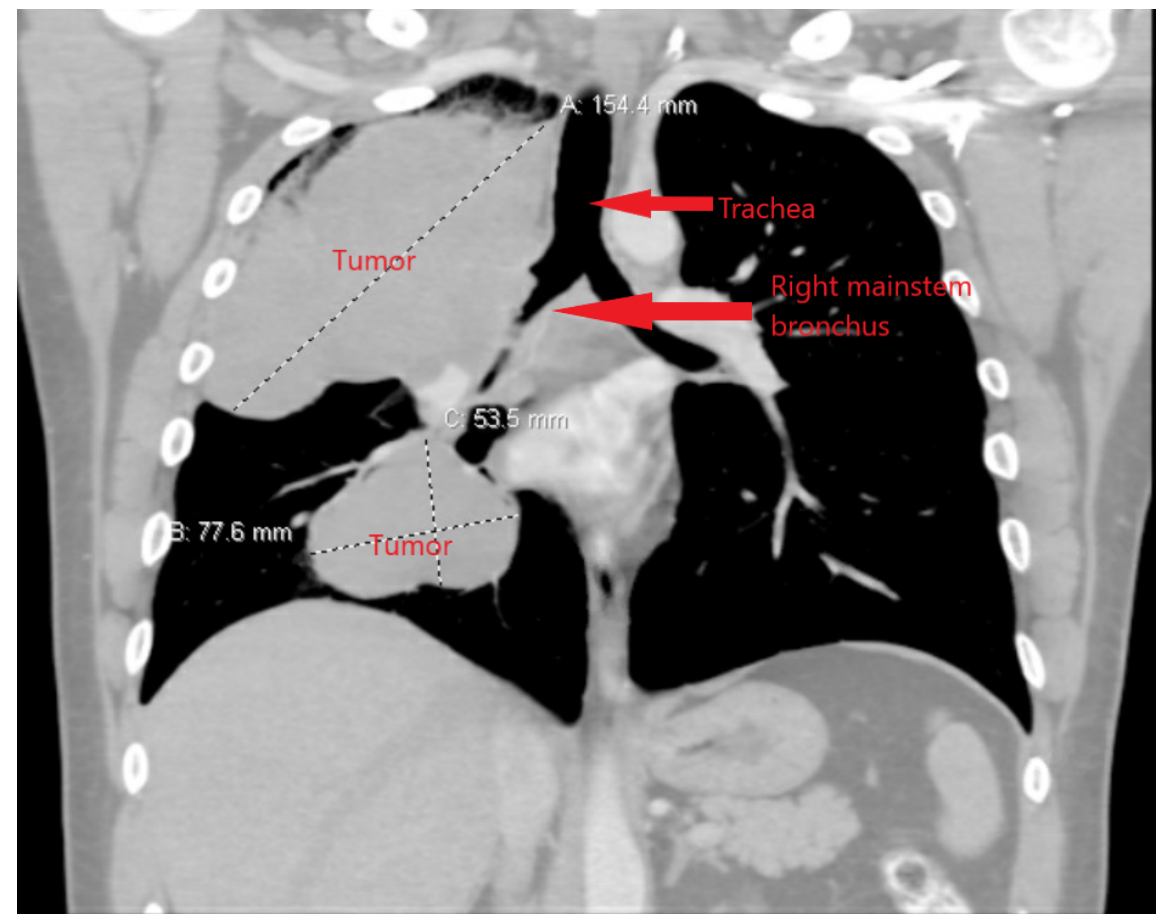




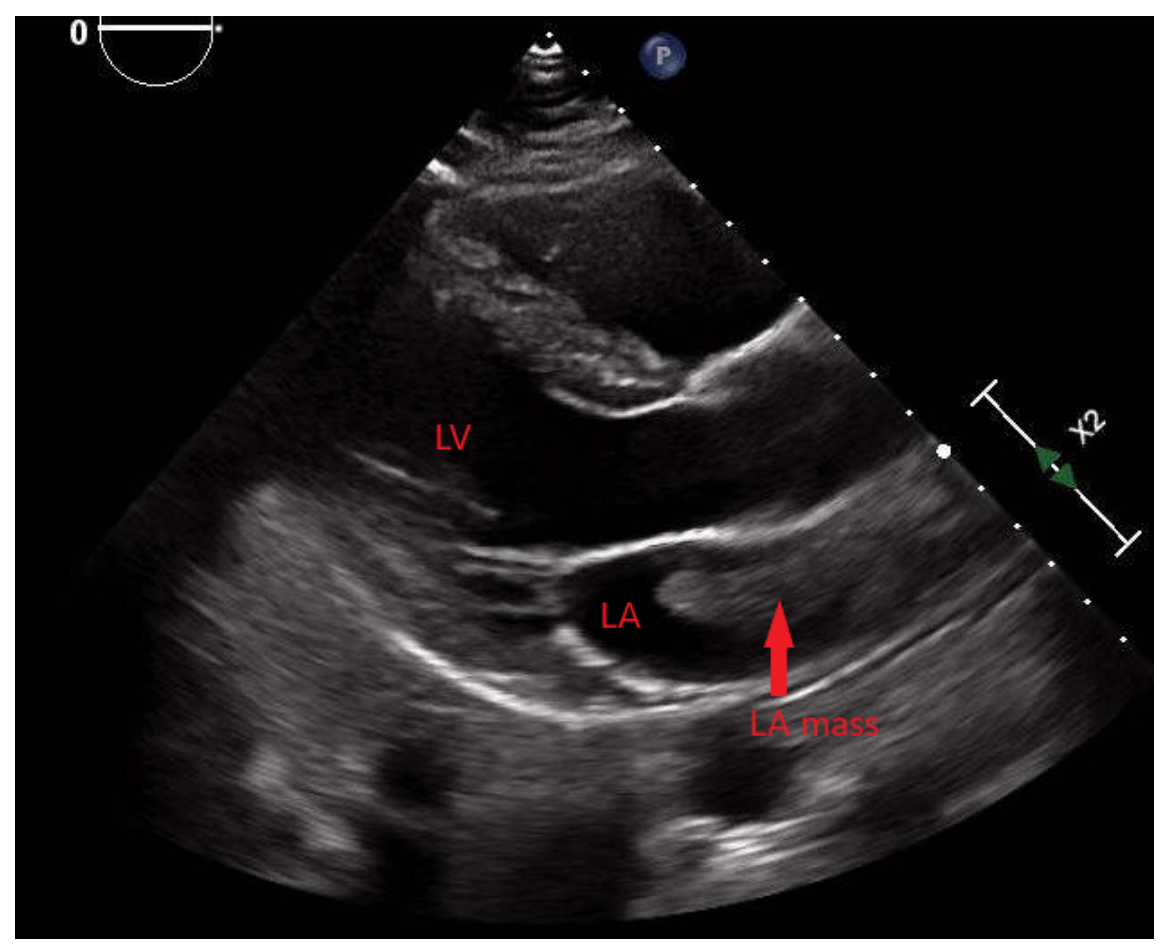

